\title{
Leitura e transferência de aprendizagem de língua estrangeira ${ }^{1}$
}

\author{
Rosângela Guimarães Seba \\ Instituto Federal do Espírito Santo - Campus de Aracruz \\ rosangelaseba@gmail.com \\ Sávio Silveira de Queiroz \\ Universidade Federal do Espírito Santo \\ savio.queiroz@ufes.br
}

\begin{abstract}
Resumo
Apesar de a literatura reconhecer a importância da transferência para a aprendizagem de língua estrangeira, poucos pesquisadores brasileiros têm dedicado seus estudos a esse fenômeno. Este estudo de caso qualitativo e exploratório buscou investigar os processos de aquisição e de transferência de estratégias de aprendizagem de alunos universitários brasileiros no contexto de leitura em Inglês para Fins Acadêmicos. Os dados, analisados interpretativamente, foram coletados em quatro estágios, por meio de questionários/inventários, diário de campo, atividades avaliativas de compreensão textual, justificativas dos participantes para o uso (ou não) de certas estratégias, e relatórios finais. Os resultados indicaram que a transferência de certas estratégias foi efetiva, favorecendo positivamente a compreensão textual em inglês. Fundamentado na teoria psicogenética de Piaget (1995, 1978), no conceito de transferência de Haskell (2001) e nas estratégias de aprendizagem de Oxford (1990), o estudo pretende contribuir para uma melhor compreensão dos processos de construção e transferência do conhecimento como resultado da interação do leitor com o texto a partir da tomada de consciência das estratégias de aprendizagem de inglês como língua estrangeira.
\end{abstract}

Palavras-chave: Aprendizagem de língua estrangeira. Leitura. Tomada de consciência. Transferência de aprendizagem. Estratégias de aprendizagem.

\footnotetext{
${ }^{1}$ Este artigo é um recorte da tese de Doutorado da primeira autora defendida em 11/12/2012, sob orientação do segundo autor.
} 


\section{Abstract}

Although recognized in the literature the importance of transfer for foreign language learning, few Brazilian researchers have dedicated their studies to this phenomenon. This qualitative, exploratory case study aimed to investigate the processes of language learning strategy acquisition and transfer of Brazilian university students in the context of English for Academic Purposes. Data, analyzed interpretatively, were collected in four stages, by means of questionnaires/inventories, field diary, reading comprehension activities, justifications (for the use or not of certain strategies) and final reports. Based on Piaget's Genetic Epistemology (1995, 1978, 1970), on Haskell's (2001) concept of transfer and on Oxford's (1990) learning strategies, the study can contribute to a better understanding of the processes of knowledge construction and transfer as a result of the interaction between reader and text, built from the grasp of consciousness of English as a foreign language learning strategies.

Keywords: Foreign language learning. Reading. The grasp of consciousness. Learning transfer. Learning strategies.

\section{Introdução}

Um dos mais fascinantes aspectos do desenvolvimento humano é a capacidade de transferir conhecimento. A transferência, ou generalização - "é responsável pelas mais simples das ideias e pelas mais altas realizações da humanidade" (HASKELL, 2001, p.23). Ela não se apresenta apenas como um veículo para uma aprendizagem futura, mas é também importante para o desenvolvimento de outras atividades cognitivas, como por exemplo, a resolução de problemas, a tomada de decisões, a metacognição, além do pensamento e da razão (LEBERMAN et.al, 2006, p.3).

$\mathrm{Na}$ aprendizagem de língua estrangeira (doravante L2), a transferência é uma questão fundamental não apenas para a formação acadêmica do indivíduo, mas, acima de tudo, para sua vida social e profissional, na qual seus conhecimentos deverão ser aplicados. Sob essa ótica, a transferência de aprendizagem é de significativa importância no contexto de Inglês para Fins Acadêmicos (English for Academic Purposes - EAP, uma modalidade da Abordagem Instrumental para Fins Específicos), uma vez que o objetivo desse curso é auxiliar o aluno a desenvolver sua capacidade de ler e de 
compreender textos acadêmicos autênticos em inglês, em um curto período de tempo, a fim de que possa aplicar esse conhecimento em outras disciplinas do programa e, principalmente, em diversos ambientes.

Defendemos ser preciso estimular o aprendiz a agir, a buscar soluções para seus próprios problemas, para que a sua aprendizagem (e a transferência do conhecimento adquirido) seja efetiva. No nosso entender, levar o aluno a se tornar mais independente significa proporcionar a ele oportunidades para a tomada de consciência das suas ações. Compreendemos, porém, o processo de tomada de consciência à luz da Epistemologia Genética de Piaget (1995, 1970), como um processo construtivo complexo, que não se reduz apenas a uma iluminação repentina ou insight, mas que evolui gradativamente do "saber fazer" ao compreender "como fazer", o que possibilita ao sujeito o planejamento, a antecipação e o controle da ação.

No contexto da aprendizagem de língua estrangeira, estudos têm demonstrado que a tomada de consciência das estratégias de aprendizagem favorece o processamento de leitura, uma vez que permite ao aluno/leitor refletir sobre suas ações, procurando explicálas, justificá-las, corrigi-las e, até mesmo, antecipá-las, enquanto busca uma solução para suas dificuldades de compreensão textual na línguaalvo (SEBA, 2008; KODA, 2004; CHAMOT, 2004).

Experiências em salas de aula e até os resultados do programa "Ciências sem Fronteiras" indicam que grande parte dos estudantes universitários brasileiros não é capaz de ler e compreender textos acadêmicos autênticos em língua inglesa, apesar de terem estudado inglês no ensino médio e de muitos terem concluído o curso de Inglês em institutos particulares de idiomas. Esse fato parece indicar que as dificuldades encontradas na compreensão textual em L2 podem estar relacionadas ao pouco conhecimento da língua-alvo, desconhecimento e/ou uso inapropriado das estratégias de leitura em língua estrangeira; incapacidade de transferir as estratégias de leitura utilizadas na língua materna para a L2 ou, ainda, segundo Alderson (1984), ao conjunto desses fatores.

Embora as pesquisas no campo de L2 tenham sido extremamente produtivas nas últimas décadas, ainda não há consenso na literatura sobre como a aprendizagem de uma segunda língua ocorre (MEISEL, 2011) e, consequentemente, como o indivíduo se torna um 
bom leitor em inglês (LI \& CHUN, 2012; KODA, op.cit.). Da mesma maneira, apesar de a importância da transferência da aprendizagem de língua estrangeira para além da sala de aula ser reconhecida, não foram encontrados estudos que tratam desse tema na literatura nacional (cf. SEBA \& QUEIROZ, 2011). Comprovada esta lacuna na literatura, justifica-se, assim, a realização da pesquisa relatada no presente artigo, cujo objetivo foi o de investigar a transferência de estratégias de aprendizagem de alunos universitários brasileiros no contexto de leitura para fins acadêmicos.

Para melhor compreensão do fenômeno da transferência, apresentamos abaixo a definição, algumas teorias principais, os tipos de transferência e os fatores que contribuem para a transferência da aprendizagem.

\section{O processo de transferência de aprendizagem}

Para uma maior compreensão da evolução do conceito de transferência, colocamos resumidamente algumas dessas principais teorias que causaram mais impacto nos estudos sobre o tema:

1. "Teoria da Disciplina Formal" também conhecida como “Teoria da Disciplina Mental", atribuída a HERBART (17761841). O cérebro humano é visto como "um grande músculo" e o seu fortalecimento dependente do intenso treinamento das faculdades mentais (raciocínio, memória, atenção, por exemplo). A transferência depende de repetições sistemáticas em determinadas disciplinas formais, como, por exemplo, Latim, Geometria ou Matemática. Adeptos dessa teoria acreditam que esse tipo de treinamento, por sua própria natureza, favorece a transferência da aprendizagem, uma vez que contribui para desenvolver uma espécie de "competência geral" (LARROYO, 1974).

2. "Teoria dos Elementos Idênticos" (THORNDIKE e WOODWORTH (1901 apud. HASKELL, 2001). Para haver transferência, é preciso haver um conjunto de elementos idênticos entre duas situações ou tarefas (a que foi assimilada 
anteriormente e a nova). Isso significa que a transferência é sempre específica, nunca geral. Essa teoria apresenta uma visão behaviorista-associacionista da aprendizagem, segundo a qual a transferência ocorre como resultado da repetição, em um novo contexto, de uma reação aprendida anteriormente por meio da tentativa e erro, e fixada devido a recompensas. A prática repetitiva (rote learning) é o fator que favorece a transferência.

3. "Teoria dos Princípios Gerais" ou "Teoria da Generalização" (JUDD, 1908 apud. HASKELL, op.cit.). Precursora das teorias cognitiva e metacognitiva da transferência, a Teoria dos Princípios Gerais considera que a transferência ocorre por meio da compreensão dos princípios gerais (abstratos) que regem um determinado fenômeno, os quais podem ser aplicados não apenas em situações (ou tarefas) semelhantes, mas também em situações (ou tarefas) diferentes da anteriormente aprendida. Segundo essa teoria, as características individuais do aprendiz (como por exemplo, a motivação), são fatores significativos para a transferência. Da mesma forma, a Teoria da Generalização reconhece que a transferência pode ser facilitada por processos indiretos, como, por exemplo, pelo uso de estratégias de aprendizagem.

4. "Teoria Cognitiva do Processamento da Informação" (SINGLEY \&ANDERSON, 1989). Nessa perspectiva, baseada no modelo do computador, a mente humana (computador) recebe a informação (input), processa essa informação (codificando-a e armazenando-a na memória) para ser, quando necessário, recuperada e utilizada no contexto de transferência. Assim, a transferência está relacionada à maneira como as informações são codificadas e organizadas na memória. Sob essa ótica, as situações de treinamento e transferência devem compartilhar elementos semelhantes, o que vem a favorecer a transferência simples.

5. "Teoria Psicogenética" (PIAGET, 1970). A transferência ocorre a partir do momento em que, ao se encontrar em um 
estado de desequilíbrio cognitivo (ou seja, ao se deparar com um obstáculo - uma situação nova desconhecida, por exemplo), o indivíduo busca meios, por meio da ação, de compensá-lo utilizando-se dos esquemas cognitivos que possui.

6. “Teoria Sócio-Histórica" (VYGOTSKY, 1978). O processo de transferência depende das estruturas construídas nas interações sociais, a partir das relações que os indivíduos estabelecem entre si, mediadas pela linguagem. Assim, para Vygotsky, a transferência não se refere à aplicação de um conhecimento (ou habilidade) adquirido a uma situação nova, mas à capacidade do sujeito de realizar sozinho aquilo que ele foi capaz de aprender e realizar com a ajuda do outro (por esta atividade estar além do seu nível de desenvolvimento real (efetivo).

7. "Teoria das Inteligências Múltiplas" (GARDNER, 1985). Segundo essa teoria, o ser humano dispõe de um número de inteligências distintas (espacial, linguística, lógico-matemática, musical, sinestésica, interpessoal e intrapessoal) que se manifestam, em graus e formas variados, para resolução de problemas. Essas inteligências raramente funcionam de forma isolada, embora sejam, de certa maneira, independentes umas das outras. Sob essa ótica, a transferência ocorre por meio da interligação dessas diversas inteligências.

8. "Teoria geral da transferência" (HASKELL, 2001). A transferência é vista no contexto instrucional. Para Haskell (op.cit, p.xiii), psicólogo e educador, a transferência (definida como "a aplicação da aprendizagem anterior em uma nova aprendizagem tanto em situações semelhantes como diferentes"), é a essência da aprendizagem e do próprio pensamento. Haverá transferência, segundo ele, apenas quando um novo conhecimento for adquirido; caso contrário, haverá apenas a aplicação do mesmo conhecimento em outra situação, e não a transferência. 
Por meio de uma análise dos estudos mais recentemente desenvolvidos (cf. SEBA \& QUEIROZ, 2011) conclui-se que a transferência da aprendizagem pode ser influenciada por diversos fatores (internos e/ou externos), pode ocorrer em diferentes níveis de complexidade e pode ser caracterizada de formas diferentes (positiva ou negativa). A motivação, as crenças, o interesse, as estratégias, o estilo de aprendizagem e o nível de consciência metacognitiva são alguns dos aspectos internos do aluno que afetam diretamente a transferência da aprendizagem. Segundo Skehan (1989), as diferenças individuais se apresentam como um fator crítico em qualquer situação de aprendizagem e não devem ser subestimadas. Reconhecer esses aspectos levaà compreensãodo porquê há variação no ritmo de aprendizagem, na transferência e, consequentemente, porque muitos alunos falham em alcançar a competência esperada na língua-alvo.

Em relação aos fatores externos que influenciam a transferência, destacam-se o contexto sociocultural, o tipo de abordagem pedagógica utilizada na sala de aula, o ambiente de ensino/aprendizagem, o professor, as oportunidades para transferência, entre outros. Para favorecer a transferência da aprendizagem, há consenso na literatura de que alguns procedimentos instrucionais devem ser seguidos: conscientização do processo de aprendizagem, desenvolvimento da capacidade metacognitiva, diversas oportunidades para prática reflexiva por meio de tarefas que estimulam a aprendizagem colaborativa por descoberta, e apoio e feedback do professor. Segundo Haskell (op.cit., p. xv), as estratégias de aprendizagem e heurística constituem uma teoria especial de transferência.

Pesquisas têm demonstrado que a transferência ocorre em níveis diferentes, podendo ser "simples"= quando não é necessário nenhum esforço cognitivo para a aplicação da transferência- ou "complexa" = quando o oposto acontece (LEBERMAN et. al.2006, p.4). Para promover o desenvolvimento cognitivo no contexto de aprendizagem formal (na sala de aula de L2, por exemplo), deve-se propiciar ao aluno oportunidades para que ele atinja a transferência complexa, embora reconhecendo que, como afirma Haskell (2001), alcançar esse tipo de transferência tão almejada e necessária no mundo atual ainda seja quase uma utopia. 
Quanto ao tipo, a transferência pode ser considerada "positiva" ou "negativa". Diz-se que a transferência é positiva quando o conhecimento adquirido facilita novas aprendizagens. Por exemplo, saber jogar voleibol, favorece a aprendizagem do tênis. A transferência negativa inibe novas aprendizagens.

No contexto do ensino e aprendizagem de L2, pesquisas têm se concentrado na transferência positiva e/ou negativa da língua materna para a língua-alvo. Esse processo de transferência linguística é considerado por alguns autores como uma estratégia para compensar a falta de conhecimento da língua-alvo, sendo os dois tipos de transferência (positiva e negativa) aspectos possíveis do mesmo fenômeno (KELLERMAN, 1995).

A transferência negativa, também chamada de "interferência", pode ocorrer de duas formas: proativamente (substratum transfer)= quando a aprendizagem anterior dificulta a nova aprendizagem, ou seja, quando a língua materna (ou a cultura na qual o aluno está inserido) interfere na aprendizagem de uma segunda língua-e retroativamente (borrowing transfer), quando, ao contrário, a língua dominante (materna) é influenciada por outras (ODLIN, 1989)). Pesquisas têm demonstrado que, quanto maior for o nível de proficiência do aluno na língua-alvo, maior será a transferência retroativa. Ao contrário, porém, a interferência proativa ocorre quanto menor for o conhecimento do aluno da L2. Esse tipo de transferência pode levar o aprendiz a cometer erros e, em alguns casos, à fossilização ${ }^{2}$. Isso não significa que a interferência seja prejudicial à aprendizagem. Um ponto importante a ressaltar é que a influência da língua materna, principalmente no contexto do ensino e aprendizagem de uma língua estrangeira (no qual o aluno só dispõe de oportunidade para praticar a L2 na sala de aula), é um processo natural que contribui para o desenvolvimento da interlíngua ${ }^{3}$ do aprendiz (ODLIN, op.cit.).

\footnotetext{
${ }^{2}$ Fossilização é um fenômeno linguístico que se refere aos erros e desvios no uso da L2 resultantes da interferência da língua materna que são internalizados e difíceis de serem superados (SELINKER, 1972).

3 Interlíngua é um termo cunhado por Selinker (op.cit.) para designar o sistema linguístico transitório entre a língua materna e a L2, constituindo uma linguagem própria da qual o aprendiz se utiliza para alcançar seus objetivos comunicativos.
} 
Além disso, não se pode deixar de considerar o fato de que, na aprendizagem de L2 do adulto em um contexto formal, a língua materna constitui a base de conhecimento e experiências sobre a qual o novo conhecimento será construído.

Os modelos teóricos utilizados para descrever a transferência, como demonstrado neste artigo, baseiam-se nas diversas correntes do pensamento, e revelam diferentes concepções do fenômeno. Entretanto, considerar a transferência da aprendizagem apenas sob o ponto de vista teórico, segundo Haskell (2001) tem sido historicamente um erro comum. $\mathrm{O}$ autor defende que é necessária uma integração entre a pesquisa, a teoria e a prática. Compartilhamos dessa visão e acreditamos que embora as teorias tenham trazido contribuições importantes para a literatura, a transferência da aprendizagem ainda permanece incompreendida. Dessa forma, confirma-se a necessidade de um esforço continuado para analisar a natureza da transferência e para sugerir técnicas mais eficientes para promovê-la.

A partir do pressuposto de que o ensino explícito e sistemático das estratégias de aprendizagem favorece a compreensão textual em inglês e a transferência do conhecimento adquirido para novos contextos de leitura, este estudo se propôs a responder duas questões:

1. Os participantes da pesquisa são capazes, após um período de quatro meses aproximadamente, de transferir o conhecimento adquirido durante o curso de Inglês para Fins Acadêmicos para novos contextos de leitura?

2. Se esse fato ocorre, que estratégias de aprendizagem são mais comumente transferidas?

Entende-se por conhecimento adquirido o resultado da aprendizagem das estratégias para o desenvolvimento da compreensão leitora em inglês como língua estrangeira, por meio do ensino explícito e sistemático na sala de aula, compreendido, neste estudo, como o tipo de intervenção pedagógica que visa promover a conscientização do aluno acerca das estratégias de aprendizagem que ele deve utilizar para desenvolver a sua proficiência na língua-alvo e/ou para ajudá-lo a resolver seus problemas de aprendizagem como, por exemplo, a compreensão textual.

Para responder a primeira pergunta, adotamos o conceito de transferência de aprendizagem à luz da teoria de Haskell (op.cit.), como mencionado anteriormente. Defendemos uma visão de leitura 
sob a ótica da teoria piagetiana, como um processo cognitivo complexo e dinâmico, no qual o leitor se apresenta como um sujeito ativo, (re)construtor de sentidos a partir da sua interação com o texto, por meio do uso apropriado de estratégias de aprendizagem. Acreditamos, dessa forma, que o processo de aquisição de estratégias facilitadoras da compreensão textual em língua estrangeira e, consequentemente, a sua transferência ocorrem a partir da tomada de consciência. Isso decorre da interação do sujeito com o objeto, evoluindo gradativamente do nível mais simples de regulação (no qual a ação que está sendo executada precede a compreensão) ao nível intermediário (em que ação e compreensão ocorrem paralelamente)e ao nível mais complexo, quando a tomada de consciência permite a reflexão do pensamento sobre ele próprio, permitindo elaboração de teorias que não mais dependerão do raciocínio concreto (PIAGET,1978). Nesta pesquisa, podemos considerar que os participantes alcançam esse terceiro nível pela elaboração de uma teoria prática facilitadora da aquisição de L2, normalmente baseada na reflexão sobre uma heurística (tentativas de ensaios, correções de erros e observação de acertos) desenvolvida durante o processo de aquisição.

Para responder a segunda pergunta do estudo, adotamos o conceito de estratégias de aprendizagem segundo Oxford (1990), por considerá-lo mais abrangente e apropriado para esta pesquisa: ações, procedimentos e passos (conscientes ou automáticos) que o aluno utiliza para melhorar a sua aprendizagem.

Para Oxford (op.cit.), a distinção entre estratégias não é sempre muito clara. A escolha pelo tipo de estratégia depende da natureza da tarefa, do objetivo do leitor e do estilo de aprendizagem de cada indivíduo. A autora dividiu as estratégias em dois grandes grupos (estratégias diretas e indiretas), cada um deles subdividido em outros três grupos:

1. Estratégias Diretas (que se referem diretamente ao processo de aprendizagem da L2): mnemônicas, cognitivas e de compensação

2. Estratégias Indiretas: metacognitivas, afetivas e sociais. 


\section{Método}

A pesquisa relatada é um estudo de caso exploratório, descritivo, de natureza qualitativa (YIN, 2003) realizado no Programa de Pós-Graduação em Psicologia da Universidade Federal do Espírito Santo, tendo como participantes um grupo de cinco alunos voluntários da área de Psicologia, com nível intermediário de proficiência em inglês.

A coleta dos dados foi realizada em ambiente natural, autêntico, de sala de aula de Inglês, cobrindo um período total de 60h/aula, dividido em cinco fases: 1) Etapa de seleção dos participantes; 2) Etapa de exploração (para verificar o nível de compreensão de textos em inglês e as estratégias utilizadas pelos participantes durante a leitura), 3) Etapa de intervenção; 4) Etapa de controle (logo após o término do curso) 5) Etapa de verificação (quatro meses após o curso). A comparação entre essas etapas forneceu subsídios para a análise dos dados.

A etapa de intervenção constou de um curso de EAP com carga horária total de $44 \mathrm{~h}$, enfatizando o desenvolvimento da habilidade leitora em inglês na área de Psicologia, por meio do ensino explícito e sistemático das estratégias de aprendizagem e de situações-problema, e aprendizagem por descoberta. Esse método incentivou a tomada de consciência dos alunos acerca do processamento de leitura em língua estrangeira. O curso e todos os procedimentos de coleta de dados foram conduzidos pela pesquisadora.

Os seguintes instrumentos foram utilizados durante as etapas da pesquisa: a) Questionário diagnóstico - para selecionar os participantes e traçar os seus respectivos perfis (Etapa de Seleção); b) Questionário/inventário = adaptados do inventário "The Strategy Inventory for Language Learning"- SILL (OXFORD, 1990), da Escala de Avaliação de Estratégias de Aprendizagem (SANTOS; BORUCHOVITCH, 2001 apud MUNEIRO, 2008) e da Taxonomia para Estratégias de Leitura (SARIG, 1987) (Etapas de seleção, de controle e de verificação); c) Justificativas escritas para uso (ou não) das estratégias (Etapas de controle e de verificação); d) Avaliações escritas de compreensão de textos acadêmicos autênticos em inglês da área de Psicologia (em todas as etapas da pesquisa); e) Relatórios finais dos alunos sobre o seu processo de aprendizagem (Etapa de 
controle); f) diários de campo (Etapa de intervenção) - escritos pela professora/pesquisadora e por um assistente.

\section{Resultados}

Resumindo os resultados gerais de cada etapa da pesquisa, observamos que, antes do programa de intervenção de estratégias (exploração), os participantes apresentaram, em média, um nível regular de compreensão leitora em inglês_- notas entre 6,0 e 6,9 (MAGLIANO et. al., 1999)_,embora todos tenham estudado em cursos particulares de idiomas por, no mínimo, três anos. Os dados do questionário diagnóstico indicaram que a tradução foi a estratégia mais utilizada por esses alunos, sendo o pouco conhecimento do vocabulário o maior obstáculo para a compreensão de textos em inglês, como se observa, por exemplo, nos depoimentos das participantes Rúbia ( "tento adivinhar o significado das palavras do texto, mas me sinto insegura, então, uso o dicionário para traduzir, e anoto tudo") e Roberta ("minha maior dificuldade na leitura em inglês é por causa do vocabulário - se não consigo traduzir, não entendo o texto”). Os dados apontam para o fato de que, anteriormente ao curso,os participantes da pesquisa concebiam a leitura como um processo de decodificação de palavras ou de frases isoladas, o que confirma a visão de Rumelhart (1980), segundo a qual uma das grandes dificuldades enfrentadas durante a leitura em língua estrangeira refere-se ao fato de o leitor não ativar suas habilidades cognitivas.

A etapa de intervenção destacou-se como contexto favorável para estimular a tomada de consciência dos participantes acerca das estratégias de aprendizagem, contribuindo para torná-los leitores mais ativos e eficientes em inglês. A participante Janine, por exemplo, afirma que "após o curso, me tornei mais atenta às estratégias, o que me deu maior segurança durante a leitura. Passei a organizar mentalmente o procedimento da leitura, a observar minhas dificuldades, a melhor administrar o meu tempo e a conseguir ler com atenção mesmo quando a leitura é mais difícil. Percebi que agora consigo ler melhor e com prazer". Rubia, também aponta que "o curso mefez ver minhas dificuldades e me deu meios para resolve-las antes, eu sabia que as tinha, mas não sabia sequer nomeá-las. Como 
disse anteriormente, a minha leitura ficou mais rápida; você me deu instrumentos dos quais eu sequer tinha conhecimento. Daí passei a usá-los em meu favor, e agora me sinto bem mais segura”.

Os dados revelaram que os participantes se tornaram capazes de formular e testar hipóteses sobre o texto, fazer inferências e buscar confirmações, ativar o conhecimento prévio, antecipar, manter e/ou modificar suas estratégias e explicar de forma coerente o uso de determinadas estratégias em certos contextos. Por meio do curso, eles também passaram a compreender a estrutura da língua inglesa, atentando para os aspectos semelhantes e diferentes em relação à língua portuguesa e, dessa forma, melhor compreendendo a razão de suas dificuldades. Em seu depoimento, Roberta menciona que "antes, eu estava bloqueada, não conseguia entender os textos. Agora, compreendo como funciona a gramática do inglês, como eles pensam, e vi que é diferente do português. Portanto, não adianta traduzir. Tenho que procurar pensar como eles".

Os participantes também tiveram oportunidade de monitorar sua aprendizagem e de avaliar o seu desempenho no curso. A ênfase nas estratégias sócioafetivas parece ter contribuído para a redução da inquietação e para a superação do medo de errar e de ser criticado pelos colegas, como evidenciado na fala de Rubia: "antes eu tinha medo de errar - ficava calada. Aqui no curso aprendi que quando exponho meus erros e discuto com alguém, aprendo melhor. Não tenho mais vergonha de falar".

Os resultados da etapa de controle indicaram que o curso proporcionou mudanças significativas no repertório de estratégias de aprendizagem dos participantes, destacando-se, principalmente, o uso mais frequente das estratégias metacognitivas (organizar, planejar e monitorar sua própria aprendizagem). Os dados das atividades avaliativas demonstraram um avanço no nível de compreensão leitora em inglês, passando, em média, para "muito bom" - notas entre 8,0 e 8,9 (MAGLIANO et. al., 1999).

Diante dos dados, verificamos que, em resposta à primeira pergunta desta pesquisa, os resultados daetapa de verificação apontaram para um efeito positivo da intervenção. Os participantes foram capazes de não apenas identificar seus problemas de leitura mas também de buscar formas de resolvê-los. Para isso, buscaram compreender suas estratégias e as características de cada texto, o que 
os levou a transferir certas estratégias para novos contextos de leitura eficientemente quatro meses após o curso.

Com referência à segunda questão do estudo, houve transferência mais significativa das seguintes estratégias:

1. ler o texto completo e anotar/selecionar as ideias principais de cada parágrafo;

2. observar as palavras-chave, expressões, frases, locuções, ou outros tipos de informação;

3. descobrir o significado das palavras/expressões decompondo-as em partes;

4. tentar adivinhar o significado das palavras desconhecidas;

5. deduzir o significado das palavras pelo contexto;

6. conseguir ler com atenção, mesmo quando a leitura é tediosa ou difícil;

7. identificar suas dificuldades de leitura;

8. controlar sua ansiedade durante a leitura;

9. identificar o quanto você está aprendendo durante a leitura;

10. relacionar a leitura a outros textos lidos anteriormente;

11. administrar o seu tempo de leitura;

12. parar durante a leitura para saber se está compreendendo o que leu;

13. associar palavras/expressões do texto a sinônimos ou a outras palavras/expressões conhecidas em inglês; e

14. perceber quando não entende o que lê, parar e reler.

\section{Considerações finais}

O estudo evidenciou que houve transferência de certas estratégias para novos contextos de leitura quatro meses após a intervenção. Porém, ficou claro também que conhecer as estratégias não é suficiente para melhorar a compreensão textual dos alunos em língua inglesa. Faz-se necessário que eles compreendam como, porqueequandousar essas estratégias, para que possam transferi-las_de maneira eficaz. Portanto, a tomada de consciência representa um potencial para a aquisição e transferência das estratégias de 
aprendizagem, uma vez que ela permite ao indivíduo apropriar-se dos mecanismos utilizados para realizar as ações de um determinado processo (PIAGET, 1978).

Considerando a discussão teórica e os resultados alcançados nesta pesquisa, alguns princípios orientadores podem ser destacados como contribuição para o ensino e a aprendizagem de línguas estrangeiras e, mais especificamente, para a área de EAP. Assim, para que haja transferência das estratégias de aprendizagem para novos contextos de leitura, é preciso que:

1. o ponto de partida da aprendizagem seja o próprio sujeito (e não o conteúdo a ser abordado);

2. os textos sejam autênticos e variados;

3. as tarefas sejam desafiadoras e estimulantes, favorecendo a tomada de consciência no seu nível mais elevado;

4. o ambiente da aprendizagem contribua para reduzir a ansiedade dos alunos e para estimular a aquisição de novos conhecimentos;

5. seja fornecida uma ampla base de conhecimentos;

6. a prática seja efetiva e constante; e

7. sejam respeitadas as diferenças individuais.

Considerando então que é possível aprender a transferir o conhecimento das estratégias de aprendizagem para novas situações de leitura em língua estrangeira, esperamos que, a partir deste estudo muitos outros se realizem para que esse processo seja cada vez mais compreendido e incentivado nas escolas.

\section{Referências}

ALDERSON, Charles. Reading: a reading problem or a language problem? In: ALDERSON, Charles.; URQUHART, A. H. Reading in a foreign language. London: Longman, 1984.

CHAMOT, Anna U. Issues in language learning strategy research and teaching. Electronic Journal of Foreign Language Teaching, v. 1, n. 1, p.14-26, 2004. Disponívelem: http://eflt.nus.edu.sg/v1n12004/chamot.htm. Acesso em: 12 jul. 2007. 
GARDNER, Howard. Frames of mind. New York: Basic Books, 1985.

HASKELL, Robert E. Transfer of learning: cognition, instruction and reasoning. London: Academic Press, 2001.

KELLERMAN, Eric. Cross-linguistic influence: transfer to nowhere? Annual Review of Applied Linguistics, v. 15, p. 125-150, 1995.

KODA, Keiko. Insights into second language reading: a cross-linguist approach. Cambridge: Cambridge University Press, 2004.

LARROYO, Francisco. História geral da pedagogia. 2 ed. São Paulo: Mestre Jou, 1974.

LEBERMAN, Sarah; McDONALD, Lex; DOYLE, Stephanie. The transfer of learning: participants' perspectives of adult education and training. Hampshire, UK: Gower, 2006.

LI, Jie; CHUN, Cecília K. Effects of learning strategies on student reading literacy performance. The Reading Matrix, v. 12, n. 1, p. 3038, Apr. 2012. Disponível em: http://www.readingmatrix.com/articles/april_2012/li_chun.pdf. Acesso em: jun. 2012.

MAGLIANO, Joseph P.; GRAESSER, Arthur; TRABASSO, Tom. Strategic processing during comprehension. Journal of Educational Psychology, v. 9, p. 615-629, 1999.

MEISEL, Jürgen M. First and second language acquisition: parallels and differences. Cambridge: Cambridge University Press, 2011.

MUNEIRO, Maria L. Estratégias de aprendizagem de alunos do ensino superior. Dissertação (Mestrado em Educação) - Faculdade de Educação, Universidade Estadual de Campinas, Campinas, 2008.

ODLIN, Terence. Language transfer: cross-linguistic influence in language learning. Cambridge: Cambridge University Press, 1989.

OXFORD, Rebeca L. Language learning strategies: what every teacher should know. Boston, Massachusetts: Heinle \& Heinle, 1990. 
PIAGET, Jean. Abstração reflexionante. Trad. Fernando Becker e Petronilha Beatriz Gonçalves da Silva. Porto Alegre: Artes Médicas, 1995.

EDUSP, 1978.

A tomada de consciência. São Paulo: Melhoramentos e . Epistemologia genética. Petrópolis: Vozes, 1970.

RUMELHART, David E. Schemata: the building blocks of cognition. In: SPIRO, Rand. J. The psychology of reading. New Jersey: Hillsdale, 1980. p. 33-58.

SARIG, Gissi. Comprehension-promoting strategies: the sum of the parts and the whole. [s.l.]:The Open University of Israel, 1987.

SEBA, RosângelaG. From collaborative writing to reading comprehension: a case study on focus on form through collaborative dialogue as a means to enhance reading comprehension in English.2008. 114p. Dissertação (Mestrado em Letras) - Instituto de Letras, Universidade Federal Fluminense, Niterói, RJ, 2008.

; QUEIROZ, Sávio S. de. Para além da sala de aula: retenção e transferência de estratégias de aprendizagem. Teoria $e$ Prática da Educação, v. 14, n. 1, p.89-97, jan/abr 2011.

SELINKER, Larry. Interlanguage. International Review of Applied Linguistics, v. 10, n. 3, p.209-231, 1972.

SKEHAN, Peter. Individual differences in second language learning. London: Edward Arnold, 1989.

SINGLEY, Mark K.; ANDERSON, John R. Transfer of cognitive skill. Cambridge, MA: Harvard University Press, 1989.

VYGOTSKY, Lev S. Mind in society: the development of higher psychological processes. USA: Harvard University Press, 1978.

YIN, Robert. Case study research: design and methods. 3rd ed. Thousand Oaks, CA: Sage, 2003. 
Leitura e transferência...

Submetido em: 12/04/13 Aceito em: 04/04/14

Title: Reading and foreign language learning transfer 